Proyecciones

Vol. 24, No 3, pp. 287-294, December 2005.

Universidad Católica del Norte

Antofagasta - Chile

\title{
COUNTABLE S*-COMPACTNESS IN L-SPACES
}

\author{
GUI - QIN YANG \\ Mudanjiang Teachers College, China
}

Received : September 2005. Accepted : November 2005

\begin{abstract}
In this paper, the notions of countable $S^{*}$-compactness is introduced in L-topological spaces based on the notion of $S^{*}$-compactness. An $S^{*}$-compact $L$-set is countably $S^{*}$-compact. If $L=[0,1]$, then countable strong compactness implies countable $S^{*}$-compactness and countable $S^{*}$-compactness implies countable F-compactness, but each inverse is not true. The intersection of a countably $S^{*}$-compact L-set and a closed L-set is countably $S^{*}$-compact. The continuous image of a countably $S^{*}$-compact $L$-set is countably $S^{*}$-compact. A weakly induced $L$-space $(X, \mathcal{T})$ is countably $S^{*}$-compact if and only if $(X,[\mathcal{T}])$ is countably compact.
\end{abstract}

Subjclass : $54 A 40$

Keywords : $L$-topology, $\beta_{a}$-open cover, $Q_{a}$-open cover, $S^{*}$-compactness, countable $S^{*}$-compactness. 


\section{Introduction}

The concept of compactness is one of most important concepts in general topology. The concept of compactness in [0, 1]-fuzzy set theory was first introduced by C.L. Chang in terms of open cover [1]. Goguen was the first to point out a deficiency in Chang's compactness theory by showing that the Tychonoff Theorem is false [5]. Since Chang's compactness has some limitations, Gantner, Steinlage and Warren introduced $\alpha$-compactness [3], Lowen introduced F-compactness, strong compactness and ultra-compactness [9], Liu introduced Q-compactness [7], Li introduced strong Q-compactness [6] which is equivalent to strong F-compactness in [10], and Wang and Zhao introduced N-compactness [16, 21].

In [15], Shi introduced a new notion of fuzzy compactness in $L$-topological spaces, which is called $S^{*}$-compactness. Ultra-compactness implies $S^{*}$-compactness. $S^{*}$-compactness implies F-compactness. If $L=$ $[0,1]$, then strong compactness implies $S^{*}$-compactness.

There has been many papers about countable fuzzy compactness of $L$-sets (see $[11,12,14,18,19,20]$ etc.). They were based on the concepts of N-compactness, Chang's compactness, strong compactness and Fcompactness respectively.

In this paper, based on the $S^{*}$-compactness, we shall introduce the notion of countable $S^{*}$-compactness and research its properties.

\section{Preliminaries}

Throughout this paper $\left(L, \bigvee, \wedge,^{\prime}\right)$ is a completely distributive de Morgan algebra. $X$ is a nonempty set. $L^{X}$ is the set of all $L$-fuzzy sets on $X$. The smallest element and the largest element in $L^{X}$ are denoted by $\underline{0}$ and 1 .

An element $a$ in $L$ is called prime if $a \geq b \wedge c$ implies $a \geq b$ or $a \geq c$. An element $a$ in $L$ is called co-prime if $a^{\prime}$ is a prime element [4]. The set of nonunit prime elements in $L$ is denoted by $P(L)$. The set of nonzero co-prime elements in $L$ is denoted by $M(L)$. The set of nonzero co-prime elements in $L^{X}$ is denoted by $M\left(L^{X}\right)$.

The binary relation $\prec$ in $L$ is defined as follows: for $a, b \in L, a \prec b$ if and only if for every subset $D \subseteq L$, the relation $b \leq \sup D$ always implies the existence of $d \in D$ with $a \leq d$ [2]. In a completely distributive de Morgan algebra $L$, each member $b$ is a sup of $\{a \in L \mid a \prec b\}$. In the 
sense of [8, 17], $\{a \in L \mid a \prec b\}$ is the greatest minimal family of $b$, in symbol $\beta(b)$. Moreover for $b \in L$, define $\alpha(b)=\left\{a \in L \mid a^{\prime} \prec b^{\prime}\right\}$ and $\alpha^{*}(b)=\alpha(b) \cap P(L)$.

For an $L$-set $A \in L^{X}, \beta(A)$ denotes the greatest minimal family of $A$ and $\beta^{*}(A)=\beta(A) \cap M\left(L^{X}\right)$.

For $a \in L$ and $A \in L^{X}$, we use the following notations in [15].

$$
\begin{aligned}
& A_{[a]}=\{x \in X \mid A(x) \geq a\}, \quad A_{(a)}=\{x \in X \mid a \in \beta(A(x))\}, \\
& A^{(a)}=\{x \in X \mid A(x) \not \leq a\} .
\end{aligned}
$$

An $L$-topological space (or $L$-space for short) is a pair $(X, \mathcal{T})$, where $\mathcal{T}$ is a subfamily of $L^{X}$ which contains $\underline{0}, \underline{1}$ and is closed for any suprema and finite infima. $\mathcal{T}$ is called an $L$-topology on $X$. Each member of $\mathcal{T}$ is called an open $L$-set and its complement is called a closed $L$-set.

Definition 2.1. [ $[8,17]]$ For a topological space $(X, \tau)$, let $\omega_{L}(\tau)$ denote the family of all lower semi-continuous maps from $(X, \tau)$ to $L$, i.e., $\omega_{L}(\tau)=$ $\left\{A \in L^{X} \mid A^{(a)} \in \tau, a \in L\right\}$. Then $\omega_{L}(\tau)$ is an $L$-topology on $X$, in this case, $\left(X, \omega_{L}(\tau)\right)$ is called topologically generated by $(X, \tau)$.

Definition 2.2. [[8, 17]] An $L$-space $(X, \mathcal{T})$ is called weakly induced if $\forall a \in L, \forall A \in \mathcal{T}$, it follows that $A^{(a)} \in[\mathcal{T}]$, where $[\mathcal{T}]$ denotes the topology formed by all crisp sets in $\mathcal{T}$.

Lemma 2.3. [[15]] Let $(X, \mathcal{T})$ be a weakly induced $L$-space, $a \in L, A \in$ $\mathcal{T}$. Then $A_{(a)}$ is an open set in $[\mathcal{T}]$.

Definition 2.4. [[20]] An $L$-space $(X, \mathcal{T})$ is called countably ultracompact if $\iota_{L}(\mathcal{T})$ is countably compact, where $\iota_{L}(\mathcal{T})$ is the topology generated by $\left\{A^{(a)} \mid A \in \mathcal{T}, a \in L\right\}$.

Definition 2.5. [[11]] Let $(X, \mathcal{T})$ be an $L$-space, $A \in L^{X} . A$ is called countably $\mathrm{N}$-compact if for every $a \in M(L)$, every countable $a$ R-neighborhood family of $G$ has a finite subfamily which is an $a^{-}$-Rneighborhood family of $G$.

Definition 2.6. [[19]] Let $(X, \mathcal{T})$ be an $L$-space, $G \in L^{X} . \quad G$ is called countably strong compact if for every $a \in M(L)$, every countable $a$-R-neighborhood family of $G$ has a finite subfamily which is an $a$-Rneighborhood family of $G$.

Definition 2.7. Let $(X, \mathcal{T})$ be an $L$-space, $a \in L \backslash\{0\}$ and $G \in L^{X}$. A family $\mathcal{U} \subseteq \mathcal{T}$ is called a $Q_{a^{-}}$open cover of $G$ if $a \leq \bigwedge_{x \in X}\left(G^{\prime}(x) \vee \bigvee_{A \in \mathcal{U} A(x)}\right)$ 
It is obvious that for $a \in M(L)$, the notion of $Q_{a}$-open cover in Definition 2.7 is the corresponding notion in [15].

Definition 2.8. [[12]] Let $(X, \mathcal{T})$ be an $L$-space, $G \in L^{X}$. $G$ is called countably F-compact if for any $a \in M(L)$ and for any $b \in \beta^{*}(a)$, every constant $a$-sequence in $G$ has a cluster point in $G$ with height $b$.

Definition 2.9. [[15]] Let $(X, \mathcal{T})$ be an $L$-space, $a \in M(L)$ and $G \in$ $L^{X}$. A family $\mathcal{U} \subseteq \mathcal{T}$ is called a $\beta_{a}$-open cover of $G$ if for any $x \in X$, it follows that $a \in \beta\left(G^{\prime}(x) \vee \underset{A \in \mathcal{U} A(x)}{\vee}\right)$.

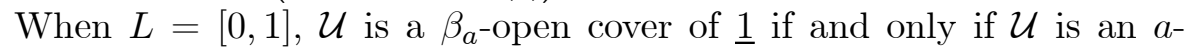
shading of $\underline{1}$ in the sense of [3]. $\mathcal{U}$ is a $\beta_{a^{\text {-open }}}$ cover of $G$ if and only if $\mathcal{U}^{\prime}$ is an $a^{\prime}$-R-neighborhood family of $G$.

\section{Countable $S^{*}$-compactness}

Definition 3.1. Let $(X, \mathcal{T})$ be an $L$-space and $G \in L^{X}$. Then $G$ is called countably $S^{*}$-compact if for any $a \in M(L)$, each countable $\beta_{a}$-open cover of $G$ has a finite subfamily which is a $Q_{a}$-open cover of $G .(X, \mathcal{T})$ is said to be countably $S^{*}$-compact if $\underline{1}$ is countably $S^{*}$-compact.

Obviously we have the following theorem.

Theorem 3.2. $S^{*}$-compactness implies countably $S^{*}$-compactness.

From Theorem 3.2, we know that an $L$-set with finite support is $S^{*}$ compact. Moreover in an $L$-space $(X, \mathcal{T})$ with a finite $L$-topology, each $L$-set is $S^{*}$-compact.

Definition 3.3. Let $\mathcal{A} \subset L^{X}, G, H \in L^{X}$ and $a \in M(L)$.

(1) $H$ is called $Q_{a}$-nonempty in $G$ if there exists $x \in X$ such that $G(x) \wedge A(x) \not \subset a^{\prime}$.

(2) $H$ is called weak $Q_{a}$-nonempty in $G$ if there exists $x \in X$ such that $a^{\prime} \notin \alpha(G(x) \wedge A(x))$.

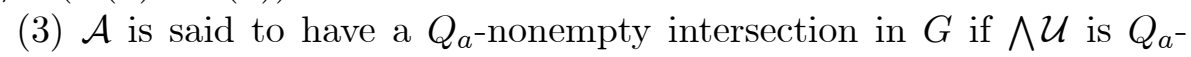
nonempty in $G$.

(4) $\mathcal{A}$ is said to have a weak $Q_{a}$-nonempty intersection in $G$ if $\wedge \mathcal{U}$ is weak $Q_{a}$-nonempty in $G$.

(5) If each finite subfamily of $\mathcal{A}$ has $Q_{a}$-nonempty intersection in $G$, then $\mathcal{A}$ is said to have finite $Q_{a}$-intersection property in $G$.

It is obvious that if $\mathcal{A}$ has a $Q_{a}$-nonempty intersection in $G$, then it also has a weak $Q_{a}$-nonempty intersection in $G$.

It is easy to prove the following theorem. 
Theorem 3.4. For an $L$-space $(X, \mathcal{T})$ and $G \in L^{X}$, the following conditions are equivalent:

(1) $G$ is countably $S^{*}$-compact.

(2) Each countable family of closed $L$-sets with finite $Q_{a}$-intersection property in $G$ has weakly $Q_{a}$-nonempty intersection in $G$.

(3) For each decreasing sequence $F_{1} \supset F_{2} \supset \cdots$ of closed $L$-sets which are $Q_{a}$-nonempty in $G,\left\{F_{i} \mid i=1,2, \cdots\right\}$ has a weakly $Q_{a}$-nonempty intersection in $G$.

Theorem 3.5. If $G$ is countably $S^{*}$-compact and $H$ is closed, then $G \wedge H$ is countably $S^{*}$-compact.

Proof. Suppose that $\mathcal{U}$ is a countable $\beta_{a}$-open cover of $G \wedge H$. Then $\mathcal{U} \cup\left\{H^{\prime}\right\}$ is a countable $\beta_{a}$-open cover of $G$. By countable $S^{*}$-compactness of $G$, we know that $\mathcal{U} \cup\left\{H^{\prime}\right\}$ has a finite subfamily $\mathcal{V}$ which is a $Q_{a}$-open cover of $G$. Take $\mathcal{W}=\mathcal{V} \backslash\left\{H^{\prime}\right\}$. Then $\mathcal{W}$ is $Q_{a^{-}}$open cover of $G \wedge H$. This shows that $G \wedge H$ is countably $S^{*}$-compact.

Theorem 3.6. If $G$ is countably $S^{*}$-compact in $\left(X, \mathcal{T}_{1}\right)$ and $f:\left(X, \mathcal{T}_{1}\right) \rightarrow$ $\left(Y, \mathcal{T}_{2}\right)$ is continuous, then $f_{L}(G)$ is countably $S^{*}$-compact in $\left(Y, \mathcal{T}_{2}\right)$.

Proof. Let $\mathcal{U} \subseteq \mathcal{T}_{2}$ be a countable $\beta_{a}$-open cover of $f_{L}(G)$. Then for any $y \in Y$, we have that $a \in \beta\left(f_{L}(G)^{\prime}(y) \vee \bigvee_{A \in \mathcal{U}} A(y)\right)$. Hence for any $x \in X, a \in \beta\left(G^{\prime}(x) \vee \bigvee_{A \in \mathcal{U}} f_{L}^{\leftarrow}(A)(x)\right)$. This shows that $f_{L}^{\leftarrow}(\mathcal{V})=$ $\left\{f_{L}^{\leftarrow}(A) \mid A \in \mathcal{U}\right\}$ is a countable $\beta_{a^{-}}$open cover of $G$. By countable $S^{*}$ compactness of $G$, we know that $\mathcal{U}$ has a finite subfamily $\mathcal{V}$ such that $f_{L}^{\leftarrow}(\mathcal{V})$ is a $Q_{a}$-open cover of $G$. By the following equation we can obtain that $\mathcal{V}$ is a $Q_{a}$-open cover of $f(G)$.

$$
\begin{aligned}
f_{\vec{L}}(G)^{\prime}(y) \vee\left(\bigvee_{A \in \mathcal{V} A(y)}\right) & =\left(\bigwedge_{x \in f^{-1}(y)} G^{\prime}(x)\right) \vee\left(\bigwedge_{A \in \mathcal{V} A(y)}^{\vee}\right) \\
& =\bigwedge_{x \in f^{-1}(y)}\left(G^{\prime}(x) \vee\left(\bigwedge_{A \in \mathcal{V} A(f(x))}^{\bigvee}\right)\right) \\
& =\bigwedge_{x \in f^{-1}(y)}(G^{\prime}(x) \vee \underbrace{\bigvee}_{A \in \mathcal{V}\left(f^{\leftarrow}(A)\right)(x)})
\end{aligned}
$$

Therefore $f_{L}(G)$ is countably $S^{*}$-compact.

Theorem 3.7. If $(X, \mathcal{T})$ is a weakly induced $L$-space, then $(X, \mathcal{T})$ is countably $S^{*}$-compact if and only if $(X,[\mathcal{T}])$ is countably compact. 
Proof. Let $(X,[\mathcal{T}])$ be countably compact. For $a \in M(L)$, let $\mathcal{U}$ be a countable $\beta_{a}$-open cover of $\underline{1}$ in $(X, \mathcal{T})$. Then by Lemma $2.2,\left\{A_{(a)} \mid A \in \mathcal{U}\right\}$ is a countable open cover of $(X,[\mathcal{T}])$. By countable compactness of $(X,[\mathcal{T}])$, we know that there exists a finite subfamily $\mathcal{V}$ of $\mathcal{U}$ such that $\mathcal{V}_{(a)}=\left\{A_{(a)} \mid\right.$ $A \in \mathcal{V}\}$ is an open cover of $(X,[\mathcal{T}])$. Obviously $\mathcal{V}$ is a $\beta_{a^{-}}$open cover of $\underline{1}$ in $(X, \mathcal{T})$, of course it is also a $Q_{a}$-open cover of 1 in $(X, \mathcal{T})$. This shows that $(X, \mathcal{T})$ is countably $S^{*}$-compact.

Conversely let $(X, \mathcal{T})$ be countably $S^{*}$-compact and $\mathcal{W}$ be a countable open cover of $(X,[\mathcal{T}])$. Then for each $a \in \beta^{*}(1), \mathcal{W}$ is a countable $\beta_{a^{-} \text {open }}$ cover of $\underline{1}$ in $(X, \mathcal{T})$. By countable $S^{*}$-compactness of $(X, \mathcal{T})$, we know that there exists a finite subfamily $\mathcal{V}$ of $\mathcal{W}$ such that $\mathcal{V}$ is a $Q_{a}$-open cover of $\underline{1}$ in $(X, \mathcal{T})$. Obviously $\mathcal{V}$ is an open cover of $(X,[\mathcal{T}])$. This shows that $(X,[\mathcal{T}])$ is compact.

Corollary 3.8. Let $(X, \tau)$ be a crisp topological space. Then $\left(X, \omega_{L}(\tau)\right)$ is countably $S^{*}$-compact if and only if $(X, \tau)$ is countably compact.

\section{A comparison of different notions of countable compact- ness}

In [13], a characterization of F-compactness was presented by means of $Q_{a}$-open cover. Analogously we can present the characterization of countable F-compactness as follows:

Lemma 4.1. Let $(X, \mathcal{T})$ be an $L$-space, $G \in L^{X}$. Then $G$ is countably F-compact if and only if for all $a \in M(L)$, for all $b \in \beta^{*}(a)$, each countable $Q_{a}$-open cover $\Phi$ of $G$ has a finite subfamily $\mathcal{B}$ such that $\mathcal{B}$ is a $Q_{b}$-open cover of $G$.

Theorem 4.2. Countable $S^{*}$-compactness implies countable F-compactness.

Proof. Let $(X, \mathcal{T})$ be an $L$-space and $G \in L^{X}$ be countably $S^{*}$ compact. To prove that $G$ is countably F-compact, suppose that $\mathcal{U}$ is a countable $Q_{a}$-open cover of $G$. Obviously for any $b \in \beta^{*}(a), \mathcal{U}$ is a countable $\beta_{b}$-open cover of $G$. By countable $S^{*}$-compactness of $G$ we know that $\mathcal{U}$ has a finite subfamily $\mathcal{V}$ which is a $Q_{b}$-open cover of $G$. By Lemma 4.1 we know that $G$ is countably F-compact.

In general, countable F-compactness needn't imply countable $S^{*}$-compactness. This can be seen from Example 6.2 in [12].

When $L=[0,1]$, since each $\beta_{a}$-open cover of $G$ is $Q_{a}$-open cover of $G$ and $\mathcal{U}$ is a $\beta_{a}$-open cover of $G$ if and only if $\mathcal{U}$ is an $a$-shading of $G$, we can obtain the following: 
Theorem 4.3. When $L=[0,1]$, countable strong compactness implies countable $S^{*}$-compactness, hence countable N-compactness implies countable $S^{*}$-compactness.

In general, countable $S^{*}$-compactness needn't imply countable strong compactness. This can be seen from Example 6.4 in [12].

Theorem 4.4. If $(X, \mathcal{T})$ is a countably ultra-compact $L$-space, then it is countably $S^{*}$-compact.

Proof. By countable ultra-compactness of $(X, \mathcal{T})$ we know that $(X, \iota(\mathcal{T}))$ is countably compact. This shows that $\left(X, \omega_{L} \circ \iota_{L}(\mathcal{T})\right)$ is countably $S^{*}$ compact from Corollary 3.8. Further from $\omega_{L} \circ \iota_{L}(\mathcal{T}) \supseteq \mathcal{T}$ we can obtain the proof.

\section{References}

[1] C. L. Chang, Fuzzy topological spaces, J. Math. Anal. Appl. 24, pp. 182-190, (1968).

[2] P. Dwinger, Characterizations of the complete homomorphic images of a completely distributive complete lattice I, Indagationes Mathematicae (Proceedings) 85, pp. 403-414, (1982).

[3] T. E. Gantner, R. C. Steinlage and R.H. Warren, Compactness in fuzzy topological spaces, J.Math. Anal. Appl. 62, pp. 547-562, (1978).

[4] G. Gierz, et al., A compendium of continuous lattices, Springer Verlag, Berlin, (1980).

[5] J. A. Goguen, The fuzzy Tychonoff theorem, J.Math. Anal. Appl. 43, pp. 734-742, (1973).

[6] Z. F. Li, Compactness in fuzzy topological spaces, Chinese Kexue Tongbao 6, pp. 321-323, (1983).

[7] Y. M. Liu, Compactness and Tychnoff Theorem in fuzzy topological spaces, Acta Mathematica Sinica 24, pp. 260-268, (1981).

[8] Y. M. Liu, M. K . Luo, Fuzzy topology, World Scientific, Singapore, (1997).

[9] R. Lowen, Fuzzy topological spaces and fuzzy compactness, J. Math. Anal. Appl. 56, pp. 621-633, (1976). 
[10] R. Lowen, A comparision of different compactness notions in fuzzy topological spaces, J. Math. Anal. Appl. 64, pp. 446-454, (1978).

[11] G. W. Meng, Countable N-compactness in L-fuzzy topological spaces, Fuzzy Systems and Mathematics. add, pp. 234-238, (1992).

[12] F.-G. Shi, G.-Q. Yang, Countable fuzzy compactness in L-topological spaces, J. Harbin Univ. Sci. \& Tech. 2, pp. 499-507, (1992).

[13] F.-G. Shi, C.-Y. Zheng, O-convergence of fuzzy nets and its applications, Fuzzy Sets and Systems 140, pp. 499-507, (2003).

[14] F.-G. Shi, Countable compactness and the Lindelöf property of L-fuzzy sets, Iranian Journal of Fuzzy Systems, 1, pp. 79-88, (2004).

[15] F.-G. Shi. A new notion of fuzzy compactness in L-topological spaces, Information Sciences 173, pp. 35-48, (205), (2005).

[16] G.-J. Wang, A new fuzzy compactness defined by fuzzy nets, J. Math. Anal. Appl. 94, pp. 1-23, (1983).

[17] G.-J. Wang, Theory of L-fuzzy topological space, Shanxi Normal University Press, Xi'an, (1988). (in Chinese).

[18] C. K. Wong, Covering properties of fuzzy topological spaces, J. Math. Anal. Appl. 43, (1973), pp. 697-704.

[19] L. X. Xuan, Countable strong compactness and strong sequential compactness, J. Nanjing Normal Unifersity 2, pp. 14-19, (1989).

[20] L. X. Xuan, Countable ultra-compactness and ultra-sequential compactness, J. Mathematical Research and Exposition 9, pp. 519-520, (1989)

[21] D. S. Zhao, The N-compactness in L-fuzzy topological spaces, J. Math. Anal. Appl. 128, pp. 64-70, (1987).

\section{Yang Gui-Qin}

Department of Mathematics

Mudanjiang Teachers College

Mudanjiang 157012

P. R. China

e-mail : guiqin_yang@hotmail.com 